\title{
Transcriptomic analysis identifies upregulation of secreted phosphoprotein 1 in silicotic rats
}

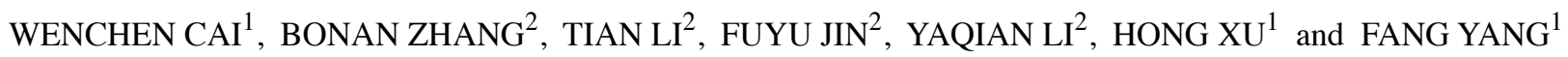 \\ ${ }^{1}$ School of Public Health, Hebei Key Laboratory for Organ Fibrosis Research; ${ }^{2}$ Basic Medicine College, \\ North China University of Science and Technology, Tangshan, Hebei 063210, P.R. China
}

Received May 8, 2020; Accepted February 22, 2021

DOI: $10.3892 /$ etm.2021.10011

\begin{abstract}
Silicosis is caused by exposure to crystalline silica and the molecular mechanism of silicotic fibrosis remains unclear. Therefore, the present study investigated the mRNA profiles of rats exposed to crystalline silica. RNA-sequencing techniques were used to observe differential expression of mRNAs in silicotic rats induced by chronic inhalation of crystalline silica particulates. Prediction of mRNA functions and signaling pathways was conducted using Gene Ontology (GO) and the Kyoto Encyclopedia of Genes and Genomes (KEGG) databases. Certain differentially expressed mRNAs were verified in lung tissue of silicotic rats by quantitative polymerase chain reaction (qPCR). Secreted phosphoprotein 1 (SPP1) was measured in serum from silicosis patients, lungs of silicotic rats and NR8383 macrophages treated with silica. A total of 1,338 mRNAs were revealed to be differentially expressed in silicotic rat lungs, including 912 upregulated and 426 downregulated mRNAs. In GO analysis of significant changes in mRNAs, the most affected processes were the defense response, extracellular space and chemokine activity in terms of biological process, cellular component and molecular function. In KEGG pathway analysis, dysregulated mRNAs were involved in systemic lupus erythematosus, staphylococcus aureus infection, complement and coagulation cascades, alcoholism and pertussis. qPCR demonstrated that expression of Spp1, Mmp12, Ccl7, Defb5, Fabp4 and Slc26a4 was increased in silicotic rats, while $L p o, I t \ln 1, L c n 2$ and $D l k 1$ expression was decreased. It was also found that SPP1 was increased in serum from silicosis patients, silicotic rats and silica-treated NR8383 macrophages. The expression of mRNAs was altered significantly in silicotic rats, which suggested that certain genes are novel targets for the diagnosis and treatment of silicosis.
\end{abstract}

Correspondence to: Dr Fang Yang or Dr Hong Xu, School of Public Health, Hebei Key Laboratory for Organ Fibrosis Research, North China University of Science and Technology, 21 Bohai Road, Tangshan, Hebei 063210, P.R. China

E-mail: fangyang@ncst.edu.cn

E-mail: xuhong@ncst.edu.cn

Key words: silicosis, Gene Ontology, Kyoto Encyclopedia of Genes and Genomes, mRNA, secreted phosphoprotein 1

\section{Introduction}

Chronic silica exposure may trigger macrophage alveolitis, induce myofibroblast transition and eventually progress to silicosis (1). Silicosis is an irreversible pulmonary disease and the fibrosis remains progressive even when patients are separated from the exposure to silica (2). In developing countries, silica-induced silicosis remains a major concern (3). There is no effective therapy and the molecular mechanism of silicotic fibrosis remains unclear (4).

With the development of gene chip and RNA sequencing (RNA-seq) technologies, bioinformatic analysis has served a significant role in screening candidate biomarkers and investigating the mechanisms of silicosis. Comparative RNA-seq has demonstrated that genes associated with immune cell interactions, immune cell responses and inflammation are significantly enriched in mice exposed to silica (5). Genes that regulate fibrosis, redox enzymes and metalloproteinases are upregulated in acute and chronic silicosis models (6). In vitro, macrophages (7) and lung epithelial cells (8) exposed to silica particles have indicated the roles of certain genes in immune responses and inflammatory pathways. These findings provide the basis to elucidate the molecular mechanisms of silica-induced pulmonary inflammation and fibrosis, and support research for the prevention and treatment of silicosis.

While these studies have provided insight into the mechanisms underlying chronic inhalation of silica, the majority of early studies were performed using silicotic models established by bronchial instillation and reported a limited number of genes, pathways and functions altered by silicosis $(5,6)$. The present study investigated the full spectrum of mRNA expression in changes of silicotic rats induced by chronic inhalation of silica, which closely approximates the development of silicosis in humans $(9,10)$. A large number of mRNAs were identified that were differentially expressed following chronic exposure to silica particles, including 912 genes that were upregulated and 426 genes that were downregulated. Furthermore, increased levels of secreted phosphoprotein-1 (SPP1) were identified in vivo, including the serum of silicotic patients and lungs of silicotic rats, and in silica-treated macrophages. Taken together, the results of the present study indicated that the expression of mRNAs was significantly altered in silicotic rats and suggested that certain genes are novel targets for the diagnosis and treatment of silicosis. 


\section{Materials and methods}

Rat silicosis model. The rats were housed in a temperature-control facility at $22-24^{\circ} \mathrm{C}$ and $70-75 \%$ humidity with a 12/12-h light/dark cycle and were given free access to water and food. All experimental and surgical procedures were approved by the Ethics Committee for Animal Experimentation of North China University of Science and Technology (2013-038). The silicotic model used in the present study has been well described and documented in our previous studies $(10,11)$. In brief, specific pathogen-free male Wistar rats (weight, $180 \pm 10 \mathrm{~g}$; age, 3 weeks; $\mathrm{n}=20$ ) were placed in a HOPE-MED 8050 exposure control apparatus (HOPE Industry and Trade Co., Ltd.) and inhaled pure air (control group) or $\mathrm{SiO}_{2}$ dust particles (80\% diameter between 1 and $5 \mu \mathrm{m}$; s5631; Sigma-Aldrich; Merck KGaA) at a concentration of $50 \pm 10 \mu \mathrm{g} / \mathrm{m}^{3}$. $\mathrm{SiO}_{2}$ was ground and heated at $180^{\circ} \mathrm{C}$ for $6 \mathrm{~h}$ for molding. The mass concentration of $\mathrm{SiO}_{2}$ was monitored by an integrated atmospheric sampler using the gravimetric method twice a week (10). Ten rats were randomly and equally divided into control and silicosis model groups. Silicotic rats were placed in the chamber for $3 \mathrm{~h}$ per day for 24 consecutive weeks. The rats were anesthetized by intraperitoneal injection of $1 \%$ pentobarbital sodium $(60 \mathrm{mg} / \mathrm{kg})$ and euthanized by blood collection from the abdominal aorta. Next, rat lung tissue was harvested and immediately frozen in liquid nitrogen for analyses.

Bioinformatics of $m R N A$ sequencing in rat lungs. Total RNA was extracted from lung tissue using TRIzol reagent (Invitrogen; Thermo Fisher Scientific, Inc.) and rRNA was removed using an Epicentre Ribo-Zero ${ }^{\mathrm{TM}}$ rRNA Removal kit (Illumina, Inc.). Subsequently, short fragments of RNA ( $200 \mathrm{nt})$ were prepared in fragmentation buffer and used as templates to synthesize cDNAs. Following purification, double-stranded cDNA was subjected to terminal repair. Specifically, a tail was added and the sequencing linker was ligated. Finally, a cDNA library of total RNA was obtained by PCR enrichment. Following the library being qualified, its insert size was determined using an Agilent 2200 Tape Station (Agilent Technologies, Inc.), which was then sequenced by a HiSeq 3000/HiSeq 2500 sequencing system (Illumina, Inc.).

Raw reads were filtered and aligned to the reference genome using alignment software HISAT (version 2.0.1) (12). Next, the ratio of the genome on the alignment and the distribution of reads on the chromosome were compared. Finally, high quality data (clean data) were obtained. BAM files were obtained by comparing clean data with the reference genome using Tophat2 software (version 2.1.1) (13). Genome location information corresponding to all reads was then counted and the coverage depth of sequencing data was evaluated. The sequenced BAM file was mapped to the reference rat gtf file to determine gene expression levels and reads per kilobase of transcript per million mapped reads values were used to calculate transcript expression levels (14). Differential expression analysis of mRNA was conducted by edgeR software (version 3.12) (15) using thresholds of $\log \mathrm{FC}>1$ and Q-value $<0.05$.

Gene ontology and pathway enrichment analysis. The biological functions of mRNA-associated target genes were analyzed by Gene Ontology (GO) enrichment analysis (http://www.geneontology.org). The pathway enrichment of mRNA-associated target genes was analyzed by the Kyoto Encyclopedia of Genes and Genomes (KEGG) database (version 12.0) (http://www.genome.jp/kegg/pathway.html).

Cell culture. The monocyte/macrophage NR8383 cell line was obtained from the Cell Bank of the Chinese Academy of Sciences (16). Cells were cultured in Ham's F-12K medium (L450KJ; Gibco; Thermo Fisher Scientific, Inc.), containing $20 \%$ (v/v) fetal bovine serum (SFBS; Bovogen Biologicals Pty., Ltd.), $100 \mathrm{IU} / \mathrm{ml}$ penicillin and $100 \mathrm{mg} / \mathrm{ml}$ streptomycin at $37^{\circ} \mathrm{C}$ in a humidified $5 \% \mathrm{CO}_{2}$ incubator. Cells at $70-80 \%$ confluence were treated with or without silica $\left(50 \mu \mathrm{g} / \mathrm{cm}^{2}\right)$.

ELISA. The samples were obtained from patients diagnosed at different stages at the Beidaihe sanatorium (Qinhuangdao, China). All the subjects had no serious diseases in the heart, brain, liver or kidneys, and those $>65$ years old were excluded. All the participants were male. The study period was between June 2017 and May 2018. The human experiments were approved by the Medical Ethics Committee of North China University of Science and Technology (2015-046) (17). Written informed consent was obtained from each subject to confirm their voluntary participation in the study. The study included 18 patients who were diagnosed with silicosis (mean age, $46.7 \pm 5.2$ years) by the Occupational Diseases Committee using diagnostic criteria for occupational pneumoconiosis of China (GBZ 70-2015). Nine control subjects (mean age, 48.0 \pm 7.0 years) from the same hospital were included (Table SI). The plasma concentration of human SPP1 was determined using a human ELISA kit (ARG81268; Arigo Biolaboratories Corp.). The assay minimum detectable concentration of SPP1 was $62.5 \mathrm{pg} / \mathrm{ml}$.

Immunohistochemistry (IHC). The samples were sequentially dehydrated, embedded in paraffin and cut into $4 \mu \mathrm{m}$ sections and then fixed in $4 \%$ paraformaldehyde solution for $48 \mathrm{~h}$ at room temperature. Paraffin-embedded sections of lung tissue and cells underwent antigen retrieval $\left(110^{\circ} \mathrm{C} ; 50 \mathrm{kPa} ; 10 \mathrm{mmol} / 1\right.$ citric acid-sodium buffer) for $80 \mathrm{sec}$, followed by quenching endogenous peroxidases with $3 \% \mathrm{H}_{2} \mathrm{O}_{2}$ for $15 \mathrm{~min}$ at room temperature. Sections were incubated with primary antibodies against SPP1 (1:100; cat. no. AF0227; Affinity Biosciences) overnight at $4^{\circ} \mathrm{C}$. The following day, they were incubated with goat anti-rabbit or anti-mouse secondary antibodies (cat. no. PV-6000; Beijing Zhongshan Jinqiao Biotechnology Co. Ltd.) at $37^{\circ} \mathrm{C}$ for $30 \mathrm{~min}$. Color development was performed by 3,3'-diaminobenzidine (ZLI-9018; OriGene Technologies, Inc.) and brown staining was considered as positive. Sections were mounted with neutral balsam and observed by microscopy (magnification, x400; BX53; Olympus Corporation).

Western blotting. Whole cell and lung tissue lysates were prepared using 100-200 $\mu 1$ RIPA buffer (BB-3201-1; BestBio; http://bestbio.qianyan.biz/) containing a protease inhibitor cocktail (P2714-1BTL; Sigma-Aldrich; Merck KGaA). The sample was incubated on ice for $30 \mathrm{~min}$ and the supernatant was collected following centrifugation $\left(14,000 \mathrm{x} \mathrm{g}, 20 \mathrm{~min}, 4^{\circ} \mathrm{C}\right)$. Total protein was estimated using a BCA assay kit (PQ0012; MultiSciences Multisciences (Lianke) Biotech Co., Ltd.). 
A

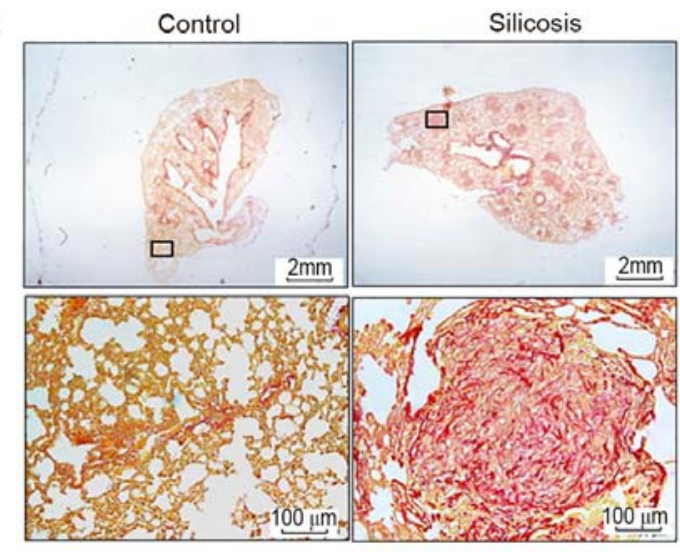

B

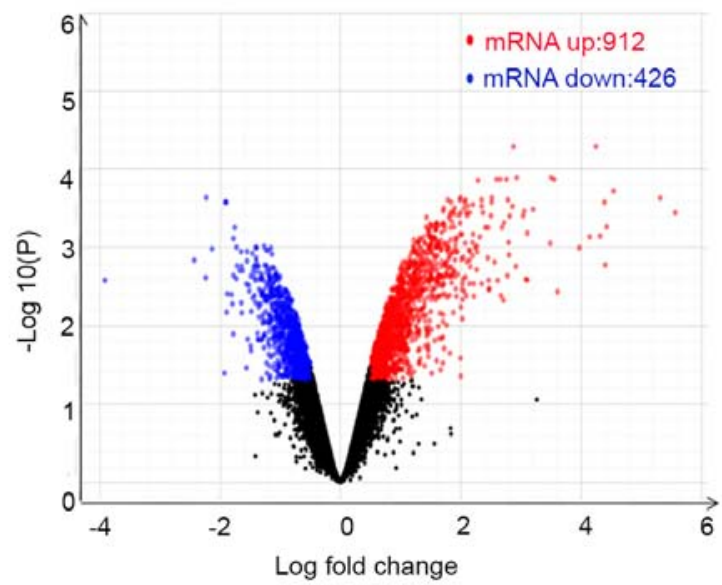

Figure 1. Volcano plot of differentially expressed mRNAs. (A) Van Gieson staining of rat lungs (scale bar=100 $\mu \mathrm{m}$ ). (B) Volcano plot of differentially expressed mRNAs.

Proteins $(20 \mu \mathrm{g} /$ lane) were separated by $10 \%$ SDS-PAGE and electrotransferred onto PVDF membranes. The membranes were blocked with $5 \%$ dry skimmed milk for $1 \mathrm{~h}$ at room temperature and then incubated overnight at $4^{\circ} \mathrm{C}$ with primary antibodies against SPP1 (1:1,000; cat. no. AF0227; Affinity Biosciences), collagen type I (Col I; 1:1000, cat. no. ab34710; Abcam) or transforming growth factor- $\beta 1$ (TGF- $\beta 1 ; 1: 1,000$; cat. no. ARG56894; Arigo Biolaboratories Corp.). The membranes were washed sequentially with TBST containing $0.2 \%$ Tween-20 and then incubated with goat anti-rabbit (cat. no. 074-1506) or anti-mouse (cat. no. 074-1806) secondary antibodies (dilution, 1:5,000; $\mathrm{KPL}$, Inc.) at $37^{\circ} \mathrm{C}$ for $1 \mathrm{~h}$. Bands were detected using the ECL $^{\mathrm{TM}}$ Prime Western blot system and average optical densities were measured using ImageJ v6.0 software (National Institutes of Health). The results were normalized against the Tub $\alpha$ expression level and corresponding control.

Quantitative polymerase chain reaction ( $q P C R)$. Total RNA was isolated from rat tissues using a RNeasy Mini-kit (Qiagen, Inc.) and quantified using a Nano-Drop-2000 spectrophotometer (Thermo Fisher Scientific, Inc.), according to the manufacturer's protocols. First-strand cDNA was generated by a reverse transcription system (cat. no. ZR102; Beijing Zoman Biotechnology Co., Ltd.), according to the manufacturer's protocols. qPCR was performed using a $2 X$ SYBR qPCR mix kit (ZF102-1; Beijing Zoman Biotechnology Co., Ltd.), under the following conditions: Initial denaturation at $94^{\circ} \mathrm{C}$ for $2 \mathrm{~min}$, followed by 40 cycles of $94^{\circ} \mathrm{C}$ for $15 \mathrm{sec}$ and $60^{\circ} \mathrm{C}$ for $30 \mathrm{sec}$ in accordance with the standard protocol of the QuantStudio ${ }^{\mathrm{TM}}$ 6 Flex Real-Time PCR System (Applied Biosystems; Thermo Fisher Scientific, Inc.). Primer sequences were as follows. GAPDH forward, 5'-GGTGAAGGTCGGTGTGAA CG-3' and reverse, 5'-CTCGCTCCTGGAAGATGGTG-3'; Dlkl forward, 5'-CAACAATGGGACTTGCGTGG-3' and reverse, 5'-TCTCGCATGGGTTAGGGGTA-3'; Lcn2 forward, 5'-CACTTCCATCCTCGTCAGGG-3' and reverse, 5'-TTC AGTTCATCGGACAGCCC-3'; Slc26a4 forward, 5'-GTG ACAATTATCGCCACCGC-3' and reverse, 5'-TGCAATAGC GTAAGCCACCA-3'; Mmp12 forward, 5'-GGAGTCCAGCCA CCAACATT-3' and reverse, 5'-TTACAGATGCAGGGAAGC
CC-3'; Sppl forward, 5'-CTTTTGCCTGTTCGGCCTTG-3' and reverse, 5'-AGCCAAGTGGCTACAGCATC-3'; Fabp4 forward, 5'-CGTAGAAGGGGACTTGGTCG-3' and reverse, 5'-CCCCACCATCCAGGGTTATG-3'; Itln1, forward, 5'-TGT TGGACTGACAATGGCCC-3' and reverse, 5'-TCCAGTGAC CTTCATGCCAG-3'; Defb5 forward, 5'-GCTGTCGCCCCT TTCTGTCT-3' and reverse, 5'-CAATCTGTCGAAAACTGC CAGG-3'; Lpo forward, 5'-GAAGGTGGGCTGTAATCC CC-3' and reverse, 5'-GAGGGAGAGTCCATCCTCGT-3'; Ccl7 forward, 5'-AGGGGTAGGAACGGTCTGTA-3' and reverse, 5'-TGAGGTCTCCAGGGCTTTAC-3'. The housekeeping gene, GAPDH, was used as the endogenous control. The results were calculated usign the $2^{-\Delta \Delta \mathrm{Cq}}$ method (18).

Statistical analysis. Statistical analysis was performed using SPSS 20.0 software (IBM Corp.). Data are presented as the mean \pm standard deviation. Two group comparisons were analyzed by the unpaired Student's t-test. The correlation between the factors was analyzed by Pearson correlation. $\mathrm{P}<0.05$ was considered to indicate a statistically significant difference.

\section{Results}

Differential expression profiles of $m R N A s$ in silicotic rat lungs. All samples in the present study were verified by histological and biochemical evidence (9-11). Fig. 1A demonstrates the formation of silicotic lesions in rats exposed to silica. In RNA-sequencing analysis, it was found that the expression of 1,338 mRNAs was changed in silicotic rats compared with that in the control group, including 912 upregulated and 426 downregulated mRNAs (Fig. 1B and Table SII). KEGG pathway analysis demonstrated that 30 pathways were enriched for the differentially expressed mRNAs, and systemic lupus erythematosus, staphylococcus aureus infection, complement and coagulation cascades, alcoholism and pertussis were the most significantly enriched KEGG pathways (Fig. 2A and Table SIII). As shown in Fig. 2B and Tables SIV-SVI, GO analysis revealed that the most significantly enriched functional terms of differentially expressed mRNAs were 
A
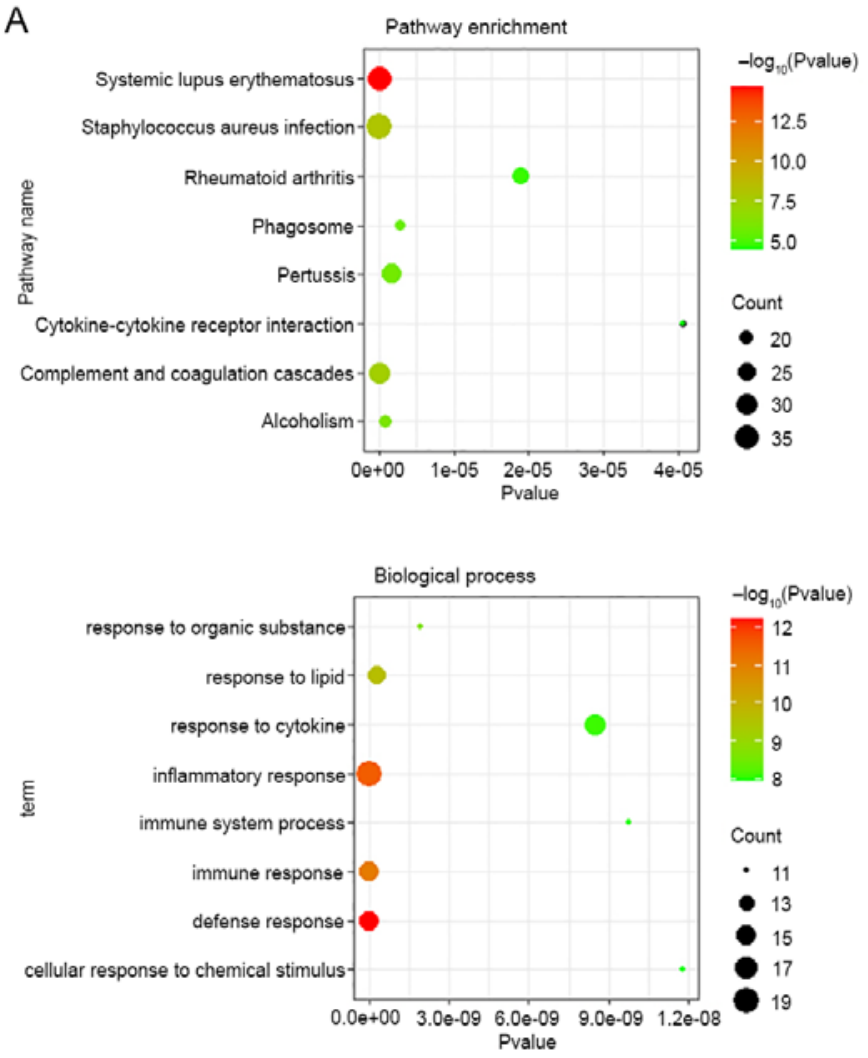

B
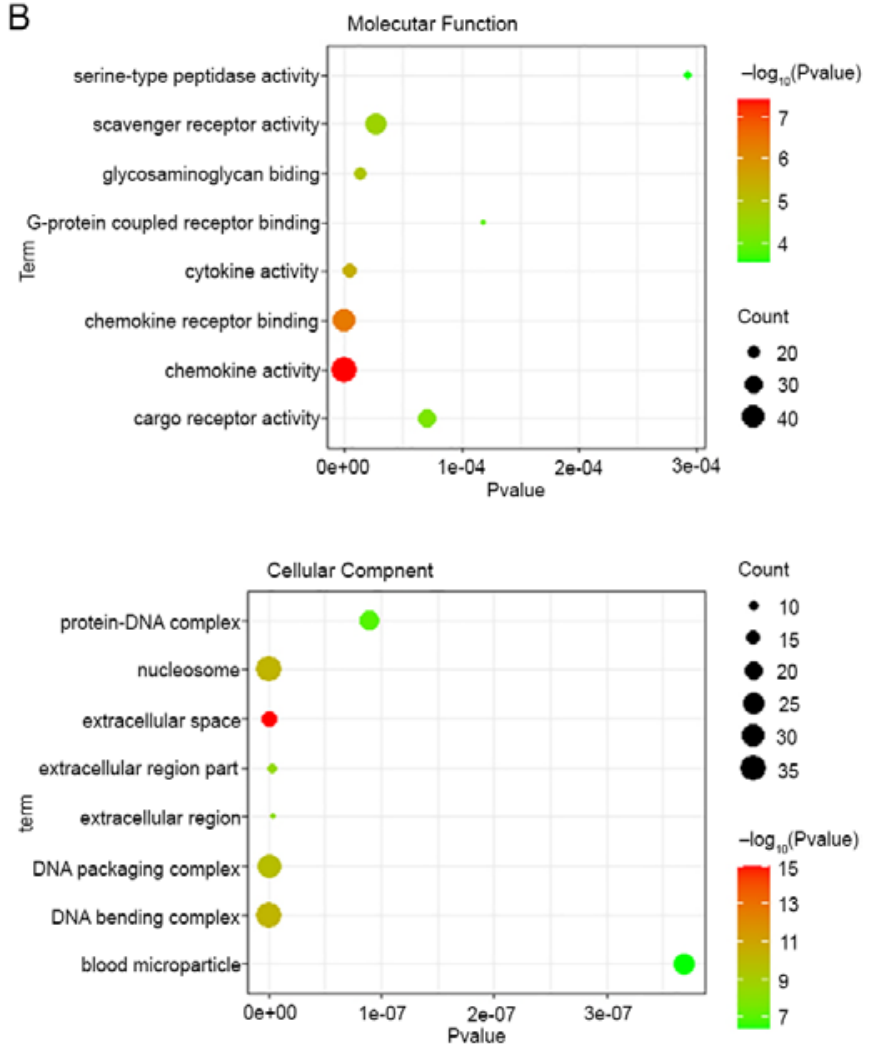

Figure 2. Bioinformatic analysis of altered mRNAs in silicotic rats. (A) Kyoto Encyclopedia of Genes and Genomes pathway assessment of differentially expressed mRNAs. (B) Gene Ontology analysis of differentially expressed mRNAs.
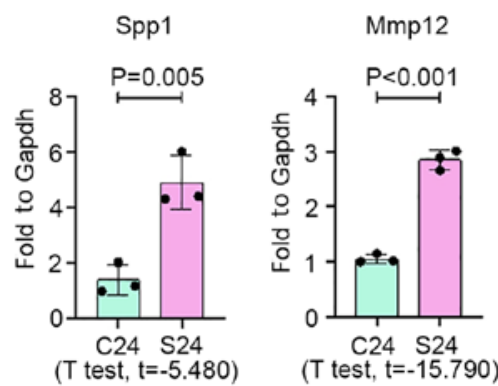

Lpo
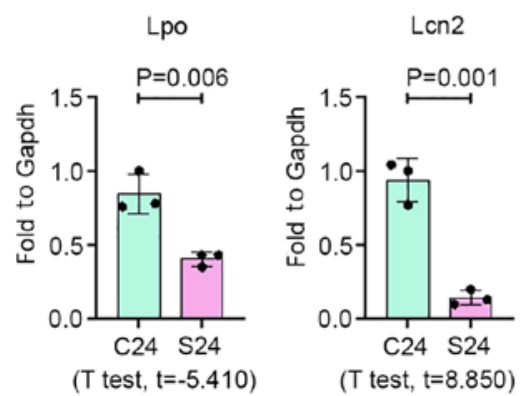
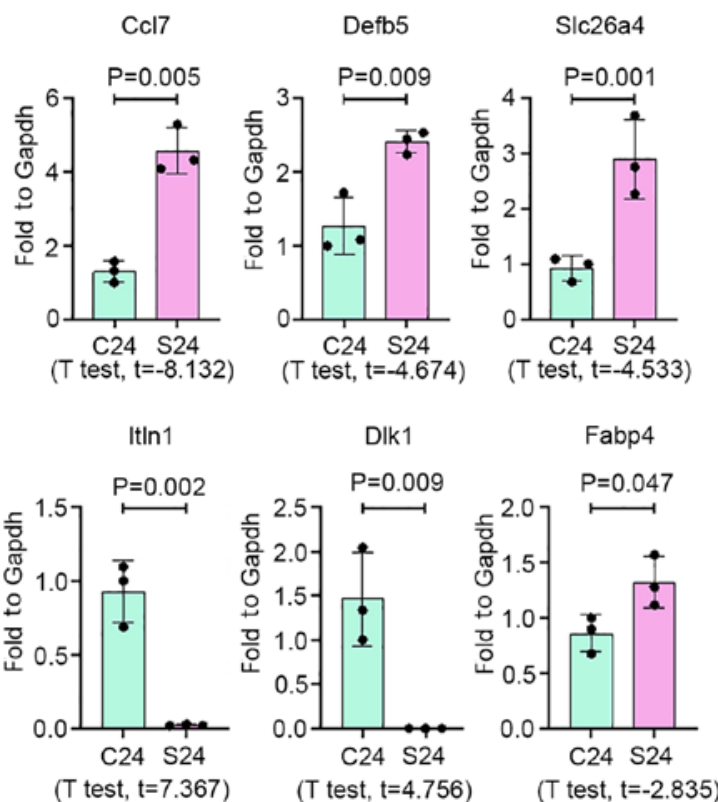

Figure 3. Validation of lung fibrosis-associated mRNAs in lung tissues. mRNA expression of Spp1, Mmp12, Ccl7, Defb5, Fabp4, Slc26a4 Lpo, Itln1, Lcn2 and $D l k l$ was detected in rats with silica-induced lung fibrosis by quantitative polymerase chain reaction. Data are presented as the mean \pm standard. $n=3$ per group.

the defense response, extracellular space and chemokine activity for classifications of biological process, and cellular component and molecular function.

Verification of differential $m R N A$ expression in silicotic rat lungs. On the basis of the fold-change and high abundance,
10 mRNAs were selected to validate the results of RNA-seq analysis in silicotic rat lungs by qPCR. As a result, expression of Spp1, Mmp12, Ccl7, Defb5, Fabp4 and Slc26a4 was increased in silicosis samples, while Lpo, Itln1, Lcn2 and Dlk1 expression was significantly decreased in silicotic lung tissues, compared with the control group (Fig. 3). 
A

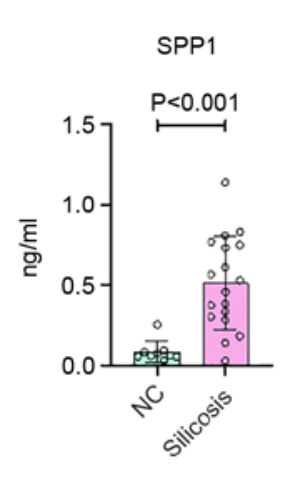

( $\mathrm{T}$ test, $\mathrm{t}=-5.959)$
B

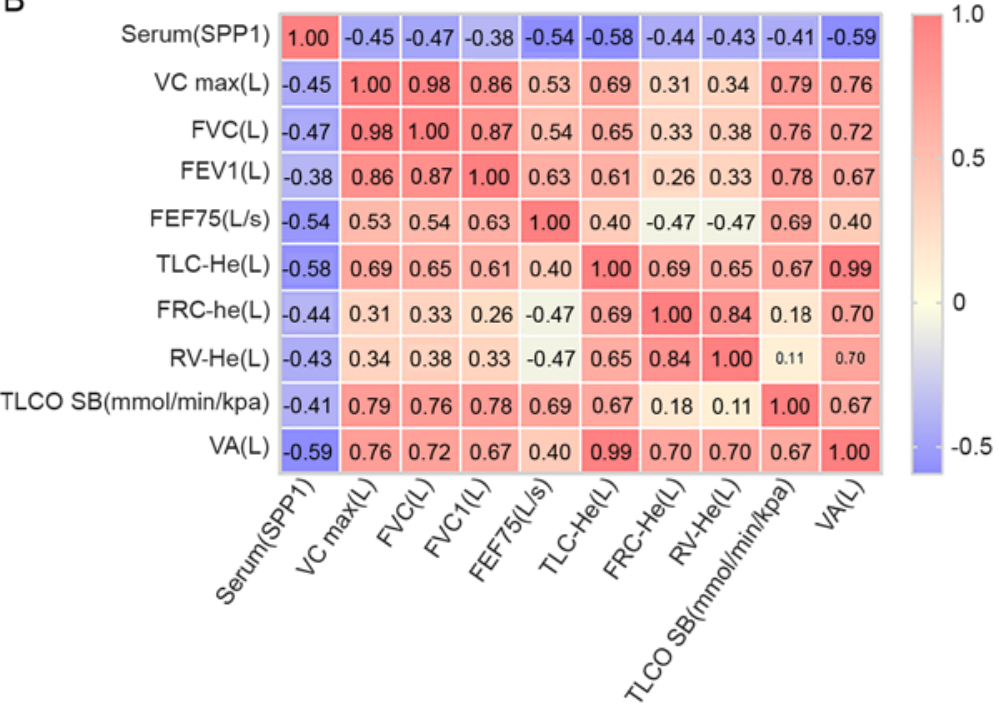

Figure 4. Expression of SPP1 in serum from silicosis patients. (A) Protein expression levels of SPP1 determined by ELISA in plasma samples from controls $(n=9)$ and patients with silicosis $(n=18)$. (B) Pearson correlation analysis of SPP1 and lung function. Data are presented as the mean \pm standard deviation. SPP1, secreted phosphoprotein 1. SPP1, secreted phosphoprotein 1; VC, vital capacity; FVC, forced vital capacity; FEV1, forced expiratory volume in the first second; FEF75, forced expiratory flow 75; TLC, total lung capacity; FRC, functional residual capacity; RV, residual volume; TLCO SB, carbon monoxide transfer factor determined in single breath; VA, alveolar ventilation.

SPP1 expression increases in serum from silicosis patients, lungs of silicotic rats and silica-treated NR8383 cells. To determine whether the mRNA findings were representative of changes in vivo and in vitro, ELISA, IHC and Western blot analyses of SPP1 in control and silicosis groups were performed, the latter of which was changed most significantly. Plasma was collected from patients with silicosis and the expression of SPP1 was analyzed by an ELISA. SPP1 expression in patients with silicosis was increased, compared with the control group (Fig. 4A and Table SVII). Pearson correlation analysis demonstrated that the expression level of SPP1 was associated with lung function (Fig. 4B). Furthermore, as shown in Fig. 5, Western blotting revealed a marked increase in the expression of SPP1 in lungs of silica-exposed rats. Furthermore, IHC staining of lungs demonstrated that the increased level of SPP1 was mostly confined to silicotic lesions of the lung, particularly in macrophages. In vitro, an increased level of SPP1 was found in silica-treated NR8383 macrophages (Fig. 6).

\section{Discussion}

Molecular understanding of silicosis has been generated from silicotic rodents with acute silicosis induced by instillation of silica. Compared with acute silicotic models, chronic inhalation of silica is a better method to investigate the complicated mechanisms of silicosis, which is relevant to human silicosis (6). In the present study, a silicotic rat model was established by chronic silica particle inhalation, which has been well documented by our group $(9,10)$, and 1,338 mRNAs were found to be altered in the silicotic group. With the progress of RNA-seq technologies, more differentially expressed mRNAs were identified than in previous reports $(19,20)$, which provides improved understanding of the development of silicosis.
To confirm the results obtained by RNA-seq analysis, 10 differentially expressed $m R N A s$ were selected for verification in silicotic lung tissues by qPCR. The results indicated that expression of Spp1, Mmp12, Ccl7, Defb5, Fabp4 and Slc26a4 was increased in silicosis samples, while expression of Lpo, Itln $1, L c n 2$ and $D l k 1$ was significantly decreased in silicotic lung tissues, compared with the control group.

An increased level of MMP-12 is a critical player in chronic pulmonary pathologies, including asthma, chronic obstructive pulmonary disease and pulmonary fibrosis (21). Overexpression of SLC26A4 was found in lungs of patients with silicosis and correlated with the onset and prognosis of silicosis (22). Although few studies have measured the expression levels of CCL7 in patients with pulmonary fibrosis, lung CCL7 expression is augmented in murine bleomycin- and radiation-induced pulmonary fibroses $(23,24)$. Additionally, CCL2 is expressed in various cell types, including macrophages, fibroblasts, endothelial cells and epithelial cells, which exhibits increased expression during silicosis (25-27). FABP4 deteriorates renal interstitial fibrosis by promoting inflammation and lipid metabolism disorders (28). Dlk1 has been reported to accelerate fibroblast-to-myofibroblast differentiation and induces myocardial fibrosis (29).

Among the upregulated mRNAs, SPPl has been demonstrated to be a marker of IPF (30). Gene or protein overexpression of SPP1 (also named osteopontin) is induced in the lungs, bronchoalveolar lavage fluid and serum of humans and rodents following exposure to various drugs and pathological agents (31). It contains an Arg-Gly-Asp motif that binds to the integrin family of adhesion molecules and is highly upregulated in mice with bleomycin-induced lung fibrosis and idiopathic pulmonary fibrosis (IPF) patients $(30,32,33)$. The present study found that Sppl had the highest false discovery rate among dysregulated mRNAs in silicotic rats. Notably, clinical correlation analyses have demonstrated that high 
A

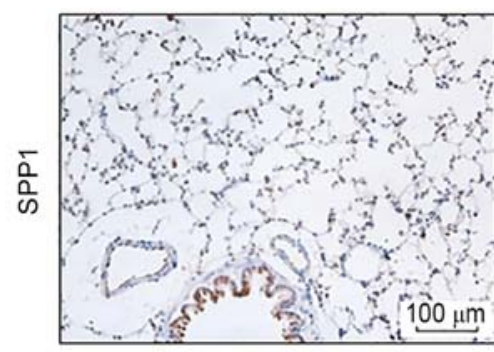

Silicosis

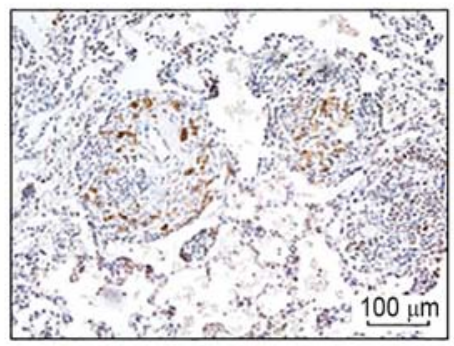

B

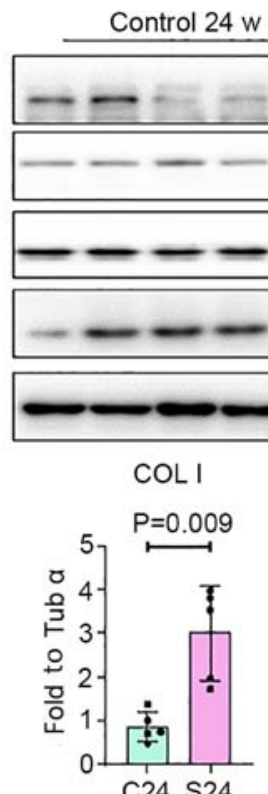

C24 S24

( $T$ test, $\mathrm{t}=-4.250$ )

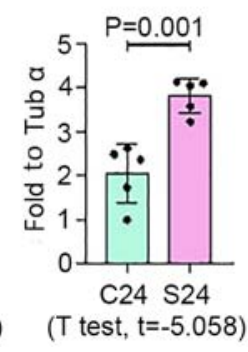

C24 S24

COL I (130 kDa)

TGF- $\beta 1(74 \mathrm{kDa})$

Tub $\alpha(55 \mathrm{kDa})$

$\operatorname{SPP} 1(60 \mathrm{kDa})$

Tub $\alpha(55 \mathrm{KD})$

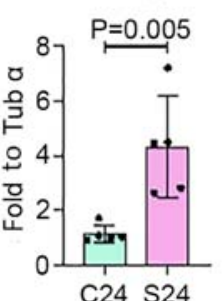

$\mathrm{C} 24 \mathrm{~S} 24$
$(T$ test, $\mathrm{t}=-3.813)$

( $T$ test, $\mathrm{t}=-5.058$ )

Figure 5. SPP1 expression is increased in silicotic rats. (A) HE and IHC staining of SPP1 in the lungs of rats (scale bar=100 $\mu \mathrm{m}$ ). (B) Protein expression of Col I, TGF- $\beta 1$ and SPP1 was detected by western blotting and quantified. Data are presented as the mean \pm standard deviation. $n=5$ per group. SPP1, secreted phosphoprotein 1; HE, hematoxylin and eosin; IHC, immunohistochemistry; Col I, collagen I; TGF, transforming growth factor.

A
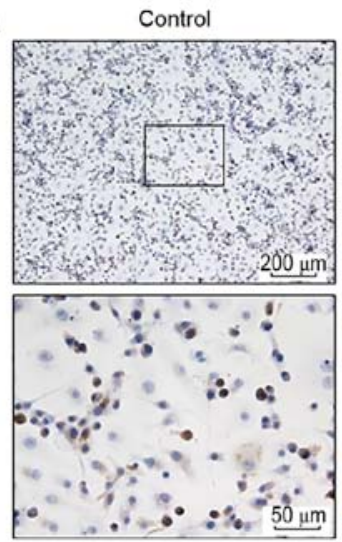

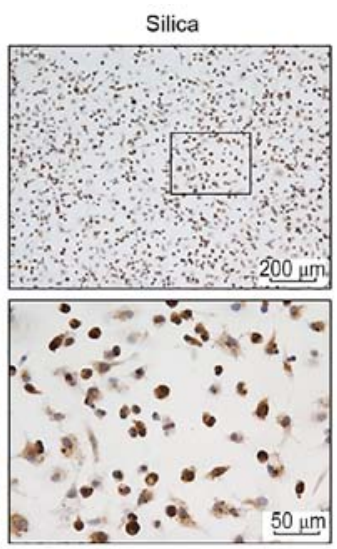

B 
promote activation of myofibroblasts (35). Furthermore, global SPP1 ablation correlates with decreased fibrosis and decreased TGF- $\beta$ in dystrophic muscle (36). Additionally, SPP1 promotes fibroblast differentiation and induces upregulation of collagen type I expression in pathological fibrosis associated with liver, skin and lung tissues (37-39). Deletion of SPP1 in bleomycin-induced lung fibrosis decreases upregulated expression of collagen type 1 and MMP2 $(40,41)$. High expression of SPP1 was also found in silica-treated macrophages. These results indicated high SPP1 expression during silicosis, which may strongly contribute toward lung fibrosis in occupational exposure to silica.

In conclusion, it was found that the expression of mRNAs was significantly altered in silicotic rats, which suggests that certain genes may be novel targets for the diagnosis and treatment of silicosis.

\section{Acknowledgements}

Not applicable.

\section{Funding}

The present study was supported by the National Natural Science Foundation of China (grant no. 81972988), Natural Science Foundation of Hebei Province (grant no. H2020209052), Science and Technology Research Project of Hebei Province universities (grant no. ZD2019077), The Science and Technology Plan Project of Tangshan City (grant no. 20130206b), and Open Fund of Key Laboratory of Functional and Clinical Translational Medicine of Xiamen Medical Collage (grant no. XMMC-FCTM201902).

\section{Availability of data and materials}

The datasets used and/or analyzed during the present study are available from the corresponding author on reasonable request.

\section{Authors' contributions}

FY and HX designed the study. WC performed all the experiments. BZ, TL, FJ and YL collected experimental samples. WC and HX interpreted the data and drafted the manuscript. FY, WC and HX confirmed the authenticity of the raw data. All authors read and approved the final version of the manuscript.

\section{Ethics approval and consent to participate}

All experimental and surgical procedures involving animals were approved by the Ethics Committee for Animal Experimentation of North China University of Science and Technology (2013-038). The human experiments were approved by the Medical Ethics Committee of North China University of Science and Technology (2015-046). Written informed consent was obtained from each subject to confirm their voluntary participation in the study.

\section{Patient consent for publication}

Not applicable.

\section{Competing interests}

The authors declare that they have no competing interests.

\section{References}

1. Li J, Yao W, Hou JY, Zhang L, Bao L, Chen HT, Wang D, Yue ZZ, Li YP, Zhang M and Hao CF: Crystalline silica promotes rat fibrocyte differentiation in vitro, and fibrocytes participate in silicosis in vivo. Biomed Environ Sci 30: 649-660, 2017.

2. Siribaddana AD, Wickramasekera K, Palipana WM, Peiris MD, Upul BK, Senevirathna KP and Dassanayake DL: A study on silicosis among employees of a silica processing factory in the Central Province of Sri Lanka. Ceylon Med J 61: 6-10, 2016.

3. Cainelli F, Tanko MN and Vento S. Silica exposure and silicosis: Action is needed now. South Med J 103: 1078, 2010.

4. Kulkarni GK: Prevention and control of silicosis: A national challenge. Indian J Occup Environ Med 11: 95-96, 2007.

5. Chen J, Yao Y, Su X, Shi Y, Song X, Xie L, You J, Tian L, Yang L, Fang $A$ and Xiong J: Comparative RNA-Seq transcriptome analysis on silica induced pulmonary inflammation and fibrosis in mice silicosis model. J Appl Toxicol 38: 773-782, 2018.

6. Langley RJ, Mishra NC, Peña-Philippides JC, Rice BJ, Seagrave JC, Singh SP and Sopori ML: Fibrogenic and redox-related but not proinflammatory genes are upregulated in Lewis rat model of chronic silicosis. J Toxicol Environ Health A 74: 1261-1279, 2011.

7. Chan JYW, Tsui JCC, Law PTW, So WKW, Leung DYP, Sham MMK, Tsui SKW and Chan CWH: RNA-Seq revealed ATF3-regulated inflammation induced by silica. Toxicology 393: 34-41, 2018.

8. Chan JYW, Tsui JCC, Law PTW, So WKW, Leung DYP, Sham MMK, Tsui SKW and Chan CWH: Profiling of the silica-induced molecular events in lung epithelial cells using the RNA-Seq approach. J Appl Toxicol 37: 1162-1173, 2017.

9. Hui Z, Dingjie X, Yuan Y, Zhongqiu W, Na M, Mingjian B, Yu G, Guangyuan L, Xuemin G, Shifeng L, et al: Silicosis decreases bone mineral density in rats. Toxicol Appl Pharmacol 348: 117-122, 2018.

10. Shifeng L, Hong X, Xue Y, Siyu N, Qiaodan Z, Dingjie X, Lijuan Z, Zhongqiu W, Xuemin G, Wenchen C, et al: Ac-SDKP increases alpha-TAT 1 and promotes the apoptosis in lung fibroblasts and epithelial cells double-stimulated with TGF-beta1 and silica. Toxicol Appl Pharmacol 369: 17-29, 2019.

11. Cai W, Xu H, Zhang B, Gao X, Li S, Wei Z, Li S, Mao N, Jin F, Li Y, et al: Differential expression of lncRNAs during silicosis and the role of LOC103691771 in myofibroblast differentiation induced by TGF- $\beta 1$. Biomed Pharmacother 125: 109980, 2020.

12. Pertea M, Kim D, Pertea GM, Leek JT and Salzberg SL: Transcript-level expression analysis of RNA-seq experiments with HISAT, StringTie and Ballgown. Nat Protoc 11: 1650-1667, 2016.

13. Kim D, Pertea G, Trapnell C, Pimentel H, Kelley R and Salzberg SL: TopHat2: Accurate alignment of transcriptomes in the presence of insertions, deletions and gene fusions. Genome Biol 14: R36, 2013.

14. Mortazavi A, Williams BA, McCue K, Schaeffer L and Wold B: Mapping and quantifying mammalian transcriptomes by RNA-Seq. Nat Methods 5: 621-628, 2008.

15. Robinson MD, McCarthy DJ and Smyth GK: edgeR: A Bioconductor package for differential expression analysis of digital gene expression data. Bioinformatics 26: 139-140, 2010.

16. Chen Y, Xu D, Yao J, Wei Z, Li S, Gao X, Cai W, Mao N, Jin F, Li Y, et al: Inhibition of miR-155-5p exerts anti-fibrotic effects in silicotic mice by regulating meprin $\alpha$. Mol Ther Nucleic Acids 19: 350-360, 2020.

17. Gao X, Xu H, Zhang B, Tao T, Liu Y, Xu D, Cai W, Wei Z, $\mathrm{Li} \mathrm{S}$, Zhang $\mathrm{H}$, et al: Interaction of $\mathrm{N}$-acetyl-seryl-aspart yl-lysyl-proline with the angiotensin-converting enzyme 2-angiotensin-(1-7)-Mas axis attenuates pulmonary fibrosis in silicotic rats. Exp Physiol 104: 1562-1574, 2019.

18. Livak KJ and Schmittgen TD: Analysis of relative gene expression data using real-time quantitative PCR and the 2(-Delta Delta C(T)) method. Methods 25: 402-408, 2001.

19. Sellamuthu R, Umbright C, Roberts JR, Young SH, Richardson D, McKinney W, Chen BT, Li S, Kashon M ans Joseph P: Molecular mechanisms of pulmonary response progression in crystalline silica exposed rats. Inhal Toxicol 29: 53-64, 2017. 
20. Sellamuthu R, Umbright C, Roberts JR, Cumpston A McKinney W, Chen BT, Frazer D, Li S, Kashon M and Joseph P: Molecular insights into the progression of crystalline silica-induced pulmonary toxicity in rats. J Appl Toxicol 33: 301-312, 2013

21. Garbacki N, Di Valentin E, Piette J, Cataldo D, Crahay C and Colige A: Matrix metalloproteinase 12 silencing: A therapeutic approach to treat pathological lung tissue remodeling? Pulm Pharmacol Ther 22: 267-278, 2009.

22. Jiang QT, Han L, Liu X and Zhu BL: Upregulation of Slc26a4 in the Early Development of Silicosis via GEO Database Analysis in vivo and in vitro. Biomed Environ Sci 32: 938-943, 2019.

23. Kaminski N, Allard JD, Pittet JF, Zuo F, Griffiths MJ, Morris D, Huang X, Sheppard D and Heller RA: Global analysis of gene expression in pulmonary fibrosis reveals distinct programs regulating lung inflammation and fibrosis. Proc Natl Acad Sci USA 97: 1778-1783, 2000.

24. Johnston CJ, Williams JP, Okunieff P and Finkelstein JN Radiation-induced pulmonary fibrosis: Examination of chemokine and chemokine receptor families. Radiat Res 157: 256-265, 2002.

25. Paine R III, Rolfe MW, Standiford TJ, Burdick MD, Rollins BJ and Strieter RM: MCP-1 expression by rat type II alveolar epithelial cells in primary culture. J Immuno 150: 4561-4570, 1993

26. Brieland JK, Jones ML, Clarke SJ, Baker JB, Warren JS and Fantone JC: Effect of acute inflammatory lung injury on the expression of monocyte chemoattractant protein-1 (MCP-1) in rat pulmonary alveolar macrophages. Am J Respir Cell Mol Biol 7: 134-139, 1992.

27. Barrett EG, Johnston C, Oberdorster G and Finkelstein JN: Antioxidant treatment attenuates cytokine and chemokine levels in murine macrophages following silica exposure. Toxicol Appl Pharmacol 158: 211-220, 1999.

28. Qiao Y, Liu L, Yin L, Xu L, Tang Z, Qi Y, Mao Z, Zhao Y, Ma $X$ and Peng J: FABP4 contributes to renal interstitial fibrosis via mediating inflammation and lipid metabolism. Cell Death Dis 10: 382, 2019.

29. Yang XQ, Zhao FF and Wang DX: Metabolism reprogramming: New insights of Dlk1 into cardiac fibrosis. Eur Heart J 40: 3574 2019.

30. Pardo A, Gibson K, Cisneros J, Richards TJ, Yang Y, Becerril C, Yousem S, Herrera I, Ruiz V, Selman M and Kaminski N: Up-regulation and profibrotic role of osteopontin in human idiopathic pulmonary fibrosis. PLoS Med 2: e251, 2005.

31. Sabo-Attwood T, Ramos-Nino ME, Eugenia-Ariza M, Macpherson MB, Butnor KJ, Vacek PC, McGee SP, Clark JC, Steele C and Mossman BT: Osteopontin modulates inflammation, mucin production, and gene expression signatures after inhalation of asbestos in a murine model of fibrosis. Am J Pathol 178 $1975-1985,2011$
32. El Deeb S, Abdelnaby R, Khachab A, Blasius K, Tingart M and Rath B: Osteopontin as a biochemical marker and severity indicator for idiopathic hip osteoarthritis. Hip Int 26: 397-403, 2016.

33. Lorenzen JM, Nickel N, Krämer R, Golpon H, Westerkamp V, Olsson KM, Haller H and Hoeper MM: Osteopontin in patients with idiopathic pulmonary hypertension. Chest 139: 1010-1017, 2011.

34. Ruberti S, Bianchi E, Guglielmelli P, Rontauroli S, Barbieri G, Tavernari L, Fanelli T, Norfo R, Pennucci V, Fattori GC, et al: Involvement of MAF/SPP1 axis in the development of bone marrow fibrosis in PMF patients. Leukemia 32: 438-449, 2018.

35. Morse C, Tabib T, Sembrat J, Buschur KL, Bittar HT, Valenzi E, Jiang Y, Kass DJ, Gibson K, Chen W, et al: Proliferating SPP1/MERTK-expressing macrophages in idiopathic pulmonary fibrosis. Eur Respir J 54: 1802441, 2019.

36. Vetrone SA, Montecino-Rodriguez E, Kudryashova E, Kramerova I, Hoffman EP, Liu SD, Miceli MC and Spencer MJ: Osteopontin promotes fibrosis in dystrophic mouse muscle by modulating immune cell subsets and intramuscular TGF-beta. J Clin Invest 119: 1583-1594, 2009.

37. Dong $\mathrm{J}$ and Ma Q: Osteopontin enhances multi-walled carbon nanotube-triggered lung fibrosis by promoting TGF-beta1 activation and myofibroblast differentiation. Part Fibre Toxicol 14: 18, 2017.

38. Hunter C, Bond J, Kuo PC, Selim MA and Levinson H: The role of osteopontin and osteopontin aptamer (OPN-R3) in fibroblast activity. J Surg Res 176: 348-358, 2012.

39. Morimoto Y, Hirahara K, Kiuchi M, Wada T, Ichikawa T, Kanno T, Okano M, Kokubo K, Onodera A, Sakurai D, et al: Amphiregulin-producing pathogenic memory T helper 2 cells instruct eosinophils to secrete osteopontin and facilitate airway fibrosis. Immunity 49: 134-150.e6, 2018.

40. Berman JS, Serlin D, Li X, Whitley G, Hayes J, Rishikof DC, Ricupero DA, Liaw L, Goetschkes M and O'Regan AW: Altered bleomycin-induced lung fibrosis in osteopontin-deficient mice. Am J Physiol Lung Cell Mol Physiol 286: L1311-L1318, 2004.

41. Takahashi F, Takahashi K, Okazaki T, Maeda K, Ienaga H, Maeda M, Kon S, Uede T and Fukuchi Y: Role of osteopontin in the pathogenesis of bleomycin-induced pulmonary fibrosis. Am J Respir Cell Mol Biol 24: 264-271, 2001.

This work is licensed under a Creative Commons Attribution-NonCommercial-NoDerivatives 4.0 International (CC BY-NC-ND 4.0) License. 